LUBLIN STUDIES IN MODERN LANGUAGES AND LITERATURE, 38(2), 2014, HTTP://WWW.LSMLL.UMCS.LUBLIN.PL

\author{
Amina Saoussany \\ Université Ibn Zohr \\ École Nationale de Commerce et de Gestion \\ BP 37/S, Hay Salam, Agadir 80000, Maroc
}

\title{
Autour de l'écrit-client : analyse de lettres commerciales de vente par correspondance produites en milieu universitaire marocain
}

\begin{abstract}
The mail order business letter has a dual purpose for the company: it arouses the customer's ordering act and builds a long term customer-relationship. This relationship is directly built on supply and is considered as a natural and legitimate action on the part of the company.

Writing a mail order business letter is not done without difficulty by the students of the Agadir National School of Commerce and Management (ENCGA). Indeed, we have noticed that despite the measures undertaken and the efforts of teachers to develop argumentative skills of learners in business French, many of them find it challenging to produce consistent and structured argumentative business letters. What are then the difficulties encountered by students during their training in economics in ENCGA in the production of professional business writings? How can we analyze their productions? How successful is argumentative competence in the French specialty? These are the questions that we have attempted to answer in this article.
\end{abstract}

1. Introduction

L'entreprise est une entité économique juridiquement autonome organisée pour produire des biens et des services pour le marché. Pour 
subsister et sauvegarder sa place dans un monde économique concurrentiel, elle doit élargir le champ de sa clientèle, diversifier ses contacts avec le monde environnant et fidéliser ses clients habituels et potentiels.

La lettre commerciale de vente par correspondance, en tant qu'outil de communication professionnelle externe, recouvre une finalité double pour l'entreprise : susciter chez le client l'acte de commande et asseoir la Relation-Client sur le long terme. Cette relation se construit en lien direct avec l'offre et s'inscrit comme une démarche naturelle et légitime de la part de l'entreprise. Elle se situe au cœur du marketing relationnel et véhicule les valeurs de persuasion, de fidélisation et de pérennisation.

La lettre commerciale de vente par correspondance doit être rédigée selon des normes bien précises. En effet, la situation d'écriture est spécifique. Les statuts et les rôles de l'émetteur et du récepteur sont déterminés : l'émetteur est un responsable au sein de l'entreprise (directeur commercial, chef de produit...) et le récepteur est un client habituel ou potentiel qu'il faut persuader de l'intérêt commercial du produit et avec qui il convient d'entretenir des relations durables. Étant essentiellement interactionnelle, cette lettre contient «une argumentation structurée sur l'offre commerciale » (Garnier \& Roussel 2007 : 92). Elle est destinée à achever de convaincre le(s) client(es) de passer commande même s'il ne faut évidemment pas négliger le poids fondamental de l'offre. Cette lettre recouvre donc une visée argumentative immédiate et reconnue par les deux acteurs $\mathrm{du}$ contrat de communication: faire acheter, et une dimension argumentative -pour reprendre et adapter la distinction introduite par Amossy (2000) - fondée sur le long terme : fidéliser la clientèle en créant un lien.

La rédaction de ce genre d'écrits ne se fait pas sans difficultés par les étudiants de l'École Nationale de Commerce et de Gestion d'Agadir (ENCGA). En effet, nous avons observé que, malgré les dispositifs mis en place et les efforts des enseignants pour développer la compétence argumentative des apprenants en français commercial, nombre d'entre eux éprouvent des difficultés à produire des lettres de 
vente cohérentes et structurées sur le plan argumentatif. Quelles sont les difficultés rencontrées par les étudiants en formation économique à l'ENCGA lors de leur production de ces écrits épistolaires professionnels ? Comment analyser leurs productions ? Comment réussir leur compétence argumentative en français de spécialité ? C'est à ces questions que nous avons tenté d'apporter des éléments de réponses dans cet article.

\section{Cadre théorique de l'étude}

Sur quels indicateurs appuyer notre analyse des lettres commerciales produites par nos étudiants? Le champ scientifique général est celui de la linguistique textuelle, discipline de base de l'analyse des textes. La théorie de la structure séquentielle d'Adam (1996) constitue pour nous un bon outil conceptuel. Compte tenu de la pertinence des modèles prototypiques et de l'éclairage qu'elle permet en termes d'hétérogénéité séquentielle, cette conception permet d'envisager les lettres de vente par correspondance en termes de structure séquentielle hétérogène qui correspond au mélange de séquences de types différents avec domination de la séquence de type argumentatif.

Si l'on soutient comme Rastier (1989) que tout texte appartient à un discours et est appréhendé à travers un genre, les apports d'Adam (1999), de Rastier (1989) et de Maingueneau (1998) nous sont utiles pour catégoriser les textes du corpus et les identifier dans leur rapport avec les pratiques sociales existantes. Les travaux de Grassi (1998) déterminent, de leur côté, la structure compositionnelle de la lettre commerciale de vente par correspondance et marquent son appartenance au genre épistolaire professionnel.

L'argumentation est la dominante de notre travail. Les apports des différentes théories de l'argumentation, celle d'Anscombre \& Ducrot (1981, 1983), de Grize (1996) et d'Amossy $(1999,2000)$ caractérisent ce qu'est l'argumentation et déterminent ses fondements logiques, théoriques et pragmatiques. L'analyse argumentative des textes s'inscrit dans différents courants de la pragmatique, fait intervenir la rhétorique ou l'art de persuader qui est revenu sur le devant de la scène avec les travaux de Perelman et Olbrechts-Tyteca (1970). Ces 
auteurs qui ont fait fructifier l'héritage de la rhétorique antique définissent la théorie de l'argumentation comme «l'étude des techniques discursives permettant de provoquer ou d'accroitre l'adhésion des esprits aux thèses que l'on présente à leur assentiment». (Traité de l'Argumentation. La nouvelle Rhétorique 1970: 5). L'argumentation développée dans les textes de nos étudiants doit avoir une visée persuasive, ce qui veut dire que les textes doivent avoir une force illocutoire afin de produire un effet sur le destinataire. Nous sommes renvoyés ici à la théorie des actes de langage mais en sachant que la force illocutoire d'un discours peut se réaliser à travers plusieurs procédés différents. L'acte illocutoire dans la lettre de vente est, en effet, un acte indirect complexe dans lequel les deux valeurs pragmatiques (constative et directive) sont simultanément actualisées. L'acte directif comprend bien évidemment la réalisation de l'acte constatif. Ces deux actes se réalisent dans la lettre de vente comme suit :

1. Transmission d'informations sur le produit

2. Invitation à l'achat (acte directif implicite).

Les deux domaines que nous avons repérés dans ce que nous avons appelé la logique argumentative sont les modes de raisonnement comme la déduction et l'explication qui enchainent des arguments à valeur de preuve. Ce sont aussi les connecteurs, en particulier les connecteurs argumentatifs.

La lettre commerciale devant présenter un nouveau produit et vanter ses qualités, il a été nécessaire d'identifier les procédés par lesquels un objet doté d'une valeur peut persuader et pousser le destinataire à désirer l'acheter. Les travaux de Catherine KerbratOrecchioni (1986) sur la subjectivité dans le langage nous ont mis sur la piste des adjectifs axiologiques, exemple super, efficace, moderne, qui présentent le produit dans une stratégie de valorisation. Le deuxième procédé concerne le texte en tant que lettre commerciale qui s'inscrit dans un dispositif énonciatif explicite : un scripteur qui établit un contact avec un destinataire. Ce dispositif obéit à des normes sociales liées aux rôles et aux statuts des deux protagonistes : c'est un 
responsable de la prospection et du marketing qui s'adresse à un client potentiel qu'il ne connait pas.

Dans l'analyse du dispositif énonciatif, nous avons isolé deux éléments. Le premier est la construction de l'image de soi par le scripteur que nous avons traitée à travers la notion d'éthos évoquée par Amossy (1999). Le deuxième est le contact établi avec le destinataire qui vise à réduire la distance avec l'autre et à renforcer l'impact de son discours. Un destinataire que l'on implique sera plus réceptif au discours d'action ou plutôt au discours d'influence qu'on lui adresse. Le texte a une fonction pragmatique qui passe par des moyens d'action sur l'autre avec l'exemple de l'usage des questions rhétoriques et de l'impératif qui incitent l'autre à faire quelque chose, ici à acheter le produit.

Nous avons accordé de l'importance à l'image du texte. Notre objectif était de vérifier s'il y avait appropriation des conventions de l'écrit épistolaire professionnel par les étudiants. Cet aspect peut être moins relié à l'argumentation, mais le découpage en paragraphes peut jouer un rôle dans la mise en valeur des arguments. De même, les mots en gras, en italique, l'usage des majuscules, ces procédés que Jean Peytard (1982) nomme entailles scripto-visuelles, sont des moyens de capter l'attention du lecteur destinataire. De façon générale, nous pouvons dire que tout le texte est traversé par une visée argumentative qui assure son homogénéité.

La relation avec le destinataire qu'on veut influencer impose cependant des limites. Ainsi, contrairement à la publicité qui se base sur l'argumentation impressive où la séduction et l'affectivité remplacent le raisonnement - on veut persuader le destinataire du message sans se soucier de la vérité -, les lettres de vente par correspondance doivent utiliser l'argumentation de façon mesurée pour rester crédibles aux yeux du destinataire. Les lettres doivent contrôler l'argumentation afin de ne pas donner l'impression au destinataire qu'on est trop insistant. Elles doivent aussi valoriser le produit avec modération. L'usage de l'hyperbole est exclu, l'excès de valorisation est malvenu. La description de l'objet doit aussi satisfaire à la condition de vérité. Les différentes dimensions de la persuasion et 
de l'argumentation qui ne sont pas faciles à articuler montrent que la lettre de vente par correspondance est un acte complexe qui implique des compétences de natures diverses que les étudiants que nous formons doivent acquérir et qu'ils doivent manifester dans la tâche qui leur a été demandée : produire une lettre commerciale de vente par correspondance en se projetant dans un rôle professionnel futur.

3. Méthodologie de recueil de données

Le public sur lequel est axée notre étude comprend des étudiants de la deuxième année de l'ENCG d'Agadir. À leur admission à l'ENCG après la réussite au TAFEM ${ }^{1}$, les étudiants étaient au nombre de 171. À leur passage en deuxième année, ils sont 150 répartis dans 6 groupes (groupes1-6), chaque groupe étant constitué approximativement de 25 étudiants.

La répartition en groupe se fait en prenant en considération les caractéristiques des apprenants (âge, série de bac, origine géographique, sexe). Chaque groupe est représentatif de l'ensemble. Il est constitué d'étudiants originaires d'Agadir, mais aussi des différentes régions du Maroc, ceci pour faciliter l'intégration entre eux et éviter le cloisonnement des apprenants originaires de la même région. Toutes les séries de bac sont également représentées dans chaque groupe, ce qui fait que l'on trouve les sciences expérimentales avec les sciences économiques, les techniques de gestion comptable et les techniques de gestion administrative dans la même classe. Pour ce qui est de l'âge, les étudiants sont tous des jeunes âgés de 18 à 21 ans. Ils ont tous le même nombre d'années d'enseignement du français (11 ans) et ils ont étudié les mêmes programmes en français au fondamental et au secondaire.

Le cours de Techniques d'Expression et de Communication en français est assuré, en deuxième année, par trois professeurs. Pour notre part, nous dispensons ce cours dans deux groupes (les groupes 3

${ }^{1}$ Le concours d'accès à l'ENCG est appelé Test d'Admissibilité à la Formation En Management (TAFEM). Il est composé d'un test écrit et d'un entretien oral. 
et 4) et nous avons en totalité 48 étudiants. Sur ces quarante-huit étudiants, seuls 28 ont participé à notre expérimentation.

Le recueil de données a été effectué principalement à travers la réalisation d'une activité d'expression écrite par les apprenants à partir d'une consigne. Cette activité concerne la production d'une lettre commerciale à dominante argumentative à propos d'un produit précis: le téléphone mobile Sumsung SGH-E330. La consigne de travail a été formulée comme suit :

L'entreprise MAROCTELECOM (SA au capital de 900000000 dhs, R.C. C7653, Code APE : 8763, Tel : 02387562, Fax : 02345680) vient de lancer sur le marché marocain sa nouvelle création dans le domaine des télécommunications : le mobile Sumsung SGH-E330. En tant que responsable chargé de la prospection et du marketing au sein de cette société, rédigez la lettre de vente par correspondance que vous allez envoyer à votre client pour lui présenter les qualités de votre nouveau produit et le persuader d'effectuer son achat.

Essayez de le convaincre en l'impliquant et en lui apportant les arguments de vente les plus ciblés.

Cette consigne est contraignante dans la mesure où elle oriente les apprenants et les guident dans leur production. En effet, l'imposition du type de texte (argumentatif), du genre (lettre professionnelle) et la précision de la situation d'écriture sont autant d'éléments qui délimitent les zones de liberté des étudiants et marquent la situation de communication en question.

Le texte demandé aux apprenants est de type argumentatif. Ce choix est justifié par le fait que la lettre de vente par correspondance est une lettre argumentative que nos étudiants rédigeront dans leur future fonction au sein de l'entreprise. A travers cet exercice, nous voulons vérifier l'appropriation des techniques argumentatives chez nos futurs cadres d'entreprise.

Le genre de discours est également spécifié : lettre commerciale de vente par correspondance. La lettre professionnelle est un écrit explicitement adressé qui se déroule en situation «non partagée » (Kerbrat-Orecchioni 1998 : 17) et qui contraint le scripteur à spécifier, grâce au paratexte (en tête, enveloppe, signature) et à certains 
éléments du texte épistolaire, sa propre identité comme celle de son/ses destinataire (s) ainsi que le cadre spatio-temporel dans lequel s'inscrit son identité d'écriture. La lettre de vente par correspondance doit être précise et claire et ne doit contenir que les arguments importants et susceptibles de persuader le client de l'intérêt commercial du produit valorisé et positivé. Les phrases doivent être courtes (10 à 15 mots au maximum) et les paragraphes concis (pas plus de six lignes). La lettre, accompagnée de pièces jointes (catalogue du produit, tarif...) ne doit pas dépasser une page dactylographiée pour ne pas lasser le destinataire.

La situation d'écriture est spécifiée : l'émetteur et le récepteur sont déterminés dans la consigne. Le scripteur est le chef du service de prospection et du marketing au sein de Maroc Télécom et le récepteur est un client susceptible d'être attiré par le produit et par conséquent d'effectuer son achat. La précision du statut et de la fonction des deux interlocuteurs se justifie par le fait que nous nous situons dans le cadre d'une communication professionnelle où les rôles doivent être bien identifiés. Etant destinés à travailler au sein de l'entreprise, les étudiants auxquels l'activité est proposée sont censés avoir conscience des spécificités de la situation de communication professionnelle (identification précise du destinataire, implication de l'énonciateur dans le discours, stratégies à mettre en œuvre pour argumenter...) en vue d'être de bons professionnels. Notre objectif est d'évaluer la compétence argumentative des étudiants dans une communication spécialisée correspondant à leur futur métier. Par cette activité, nous avons choisi de créer une vraie simulation : pousser les étudiants à se mettre à la place d'une personne ayant un statut précis au sein de l'entreprise et à rédiger une lettre argumentative à orientation publicitaire capable de convaincre l'acheteur potentiel.

Les écrits théoriques sur l'argumentation nous ont conduites à considérer quatre phases principales en interrelation constante qui sont à la base de la construction de la grille d'évaluation des lettres commerciales à visée persuasive qui constituent notre corpus. 
Tableau 1: Grille d'évaluation du corpus

\begin{tabular}{|l|l|l|}
\hline Phase 1 & $\begin{array}{l}\text { Etude de l'image } \\
\text { du texte. }\end{array}$ & $\begin{array}{l}\text { - Présentation de la lettre; } \\
\text {-Structure typographique des } \\
\text { paragraphes et leur structure } \\
\text { textuelle. }\end{array}$ \\
\hline Phase 2 & $\begin{array}{l}\text { Gestion du discours } \\
\text { argumentatif. }\end{array}$ & $\begin{array}{l}\text {-Structure compositionnelle de } \\
\text { la lettre; } \\
\text {-Mouvement argumentatif ; } \\
\text {-Relation émetteur/récepteur. }\end{array}$ \\
\hline Phase 3 & $\begin{array}{l}\text { Analyse de l'objet } \\
\text { à promouvoir. }\end{array}$ & $\begin{array}{l}\text {-L'objet présenté : cohésion } \\
\text { nominale. } \\
\text {-L'objet décrit : } \\
\text { - description objective ; } \\
\text {-description subjective. }\end{array}$ \\
\hline Phase 4 & $\begin{array}{l}\text { Etude du dispositif } \\
\text { d'énonciation. }\end{array}$ & $\begin{array}{l}\text {-Les mécanismes de prise en } \\
\text { charge énonciative ; } \\
\text {-La référence à l'énonciataire } \\
\text { dans le discours. }\end{array}$ \\
\hline
\end{tabular}

4. Analyse et interprétation des résultats

L'analyse des lettres commerciales à visée persuasive du corpus a montré que les difficultés des étudiants en argumentation écrite en communication de spécialité sont d'ordre textuel et discursif. Les problèmes locaux touchant à des micro-éléments de la phrase (morphologie, orthographe d'usage, conjugaison...) ne sont pas abordés, non que ceux-ci soient mineurs, mais ce sont des problèmes auxquels nous n'avons pas accordé attention eu égard aux objectifs de recherche que nous nous sommes fixés.

4.1. Première phase : Etude de l'image du texte 4.1.1. Présentation de la lettre

Notre corpus de lettres ne comporte pas de variantes de typographie : toutes les lettres ont été saisies au moyen de logiciel informatique de traitement de texte. Elles sont toutes de longueur normale (une page), sauf la lettre 6 qui est d'une longueur remarquable (une page et 
demie). Les lettres produites par les apprenants sont toutes compréhensibles et se définissent comme des écrits épistolaires. Elles sont immédiatement identifiables dans la mesure où elles proposent une certaine configuration normative de leur aire scripturale. Sur l'ensemble des lettres analysées, sept sont jugées bonnes selon ce critère. Elles comprennent les éléments obligatoires à l'identification du genre de façon correcte et complète. Les éléments relatifs à l'entreprise (nom, adresse, capital, statut juridique, numéro d'immatriculation au registre du commerce et la patente) sont également tous présents et situés en haut ou en bas de page selon les normes conventionnelles de présentation du courrier d'entreprise.

21 lettres sont moyennement bonnes et présentent certaines défaillances au niveau de la présentation de la zone paratextuelle. Ces maladresses sont variées. Elles concernent l'absence du destinataire, de la signature, du lieu ou de la date, la conception théorique et vague de l'objet, l'oubli des pièces jointes et l'erreur sur le lieu et/ou la date. Les scripteurs de ces lettres n'ont pas porté assez d'attention à la présentation formelle de leurs écrits, peut-être parce que le plus important pour eux était le texte. Ils étaient probablement conscients que le vrai destinataire de leur lettre était l'enseignant et non pas un acheteur potentiel.

\subsubsection{Structure textuelle des paragraphes}

La structuration des paragraphes n'est pas toujours bien maîtrisée. 18 étudiants ont bien segmenté leurs lettres contre 11. Celles-ci sont constituées de paragraphes bien délimités grâce à leur structure textuelle que l'on peut identifier facilement et à leur fonction de marqueurs de frontière de contenu. Chaque paragraphe développe une idée ou un argument en faveur du téléphone « Sumsung SGH-E330 », ce qui met en relief la construction de l'argumentation. 11 étudiants ont mal segmenté leurs productions. Ils ont présenté des lettres découpées en paragraphes qui développent plus d'une idée et qui gagneraient à être segmentés. Les découpages en paragraphes effectués par les étudiants ne correspondent pas toujours à une structuration de l'argumentation. La présentation du contenu en 
parties relativement longues qui pourraient être divisées en paragraphes témoigne de la difficulté des étudiants à comprendre la «fonction rhétorique » du paragraphe qui est celle de marqueur de frontière de contenu.

4.2. Deuxième phase : gestion du discours argumentatif 4.2.1. Structure compositionnelle de la lettre

Sur le plan général de la lettre, l'appropriation du schéma prototypique épistolaire présente quelques défaillances. En effet, si 12 lettres semblent se conformer au modèle prototypique épistolaire, des écarts par rapport à la norme épistolaire ont été constatés dans les autres lettres. Le tableau ci-dessous explicite ces écarts :

Tableau 2 : Ecarts dans l'appropriation de la structure compositionnelle de la lettre

\begin{tabular}{|l|c|c|}
\hline \multicolumn{1}{|c|}{ Ecarts constatés } & Liste des lettres & Total \\
\hline Absence de péroraison & $\begin{array}{c}3,9,11,14,16,18, \\
19,20,21,26\end{array}$ & 10 \\
\hline Absence de clôture & $8,10,23,27$ & 4 \\
\hline $\begin{array}{l}\text { Absence de péroraison et de } \\
\text { clôture }\end{array}$ & 27 & 1 \\
\hline $\begin{array}{l}\text { Absence d'ouverture, de } \\
\text { péroraison et de clôture }\end{array}$ & 15 & 1 \\
\hline
\end{tabular}

Ces écarts par rapport à la norme relèvent d'une non-maîtrise du schéma textuel prototypique du genre épistolaire. Les étudiants ont sans doute des difficultés à oublier que le vrai destinataire de leurs écrits est le professeur. Les textes ont été réalisés en situation de simulation et non pas en situation réelle. On ne sait pas si les étudiants, une fois en situation professionnelle, feraient de tels oublis.

\subsubsection{Argumentation et connecteurs :}

La fréquence de l'emploi des connecteurs argumentatifs varie dans les productions des étudiants. Le tableau suivant montre cette variation : 
Tableau 3 : fréquence des connecteurs argumentatifs dans les lettres

\begin{tabular}{|c|l|c|}
\hline $\begin{array}{c}\text { Nombre de } \\
\text { connecteurs } \\
\text { argumentatifs }\end{array}$ & \multicolumn{1}{|c|}{ Liste des lettres } & Total \\
\hline 0 & $8,12,13,15,20,21,25$ & 7 \\
\hline 1 & $1,4,5,7,10,22$ & 6 \\
\hline 2 & $2,6,11,14,17,18,23,27$ & 8 \\
\hline 3 & $319,12,16,9,28$ & 6 \\
\hline 6 & 24 & 1 \\
\hline
\end{tabular}

L'absence de connecteurs argumentatifs ne signifie pas que les lettres ne sont pas persuasives. Celles-ci sont satisfaisantes sur le plan argumentatif quoiqu'elles ne contiennent pas de connecteurs.

Les connecteurs les plus utilisés par les apprenants sont les connecteurs introducteurs d'arguments (16 occurrences) viennent ensuite les introducteurs d'arguments anti-orientés (11 occurrences). Les introducteurs de conclusion se situent au troisième rang $(8$ occurrences).

La présence de connecteurs ne signifie pas que ceux-ci soient utilisés correctement dans les lettres. L'analyse du corpus montre l'existence d'emplois erronés des connecteurs interphrastiques tels que : «ainsi, or qui ont un fonctionnement discursif qui va au-delà de la phrase »(Holtzer 2004: 58). Ces connecteurs argumentatifs qui : expriment respectivement la déduction et la concession sont utilisés par 8 étudiants pour exprimer l'addition L'exemple suivant est explicite de cette défaillance :

Ex : (lettre 18) «En effet, ce portable contient un appareil photo numérique permettant de prendre des clichés sur le vif avec des couleurs trop claires (assertion1)

Ainsi, sur ce mobile, on apprécie l'horloge analogique très chic qui s'affiche sur l'écran externe et peut même apparaître au mode veille » (assertion 2).

La relation entre les deux assertions n'est pas la déduction, la deuxième assertion n'étant pas la conséquence de la première. L'emploi du connecteur interphrastique ainsi ne convient pas. Il ne 
s'agit pas d'introduire une conclusion, mais de relier deux énoncés qui se complètent étant donné qu'ils développent chacun une qualité du téléphone portable. Un additif à la place du connecteur ainsi traduira mieux la relation logique entre les deux phrases.

Les étudiants de l'ENCG ne paraissent pas très à l'aise avec le maniement des connecteurs argumentatifs. Dans certains cas, les connecteurs manquent totalement. Dans d'autres cas, le connecteur employé est erroné, ce qui montre la difficulté des étudiants à argumenter. L'erreur dans l'emploi des connecteurs peut s'expliquer par le fait que les étudiants ne maîtrisent pas les normes de la grammaire textuelle qui assurent la structuration et l'organisation de l'unité texte, ou/et que le fonctionnement de tel ou tel connecteur n'est pas acquis. L'emploi efficace des connecteurs nécessite un enseignement qui prendra en charge les macrostructures du texte.

\subsubsection{La relation énonciative}

Pour ce qui est de la relation énonciative, la plupart des étudiants sont conscients de l'importance de l'implication du client et introduisent bien les indices d'allocution. Mais, l'impératif et les questions rhétoriques comme moyens d'action sur le client qui visent à transformer sa compétence modale en assurant le passage du SAVOIR sur les qualités du produit au VOULOIR l'acheter, sont peu utilisés. Ceci affaiblit, selon nous, le caractère interactionnel et argumentatif de la lettre.

Dans notre corpus, l'usage des questions et des impératifs se présente comme suit :

Tableau 4 : Usage des questions et des impératifs dans les lettres

\begin{tabular}{|c|c|c|}
\hline Impératifs+questions & Lettres 4et 6 & 2 lettres \\
\hline Impératifs & Lettres 1, 2, 5, 7, 8, 10, & 14 lettres \\
& $11,12,13,17,19,21$, & \\
& 22,24 & \\
\hline Questions & Lettres 23, 25 & 2 lettres \\
\hline Ni impératifs ni & Lettres 3, 9, 14, 15, 16, & 10 lettres \\
questions & $18,20,26,27,28$ & \\
\hline
\end{tabular}


Les impératifs et les questions, en tant que moyens d'action sur l'autre, sont utilisés efficacement dans deux lettres. Ils sont bien intégrés à leur développement argumentatif et renforcent leur caractère interactionnel. Les questions rhétoriques qui n'appellent pas de véritables réponses mais qui ont une fonction conversationnelle et un caractère communicationnel sont utilisées dans 4 lettres. On pourrait supposer que la presque totalité des apprenants réduit le rôle de la question à l'interrogation en vue d'obtenir des informations du destinataire. La question en tant que moyen d'action sur l'autre semble mal connue. De rares cas d'usage de cet outil linguistique sont repérés.

\subsection{Troisième phase : analyse de l'objet à promouvoir}

4.3.1. Présentation de l'objet

L'analyse du corpus montre que le produit, objet de vente, est nommé de différentes manières. Il est désigné par son nom technique « Sumsung SGH-E330 », «Le E330 », «Le SGH-E330 », mais aussi par des références nominales telles que «ce nouveau produit», «le nouveau mobile », « superbe mobile », « produit révolutionnaire », «petit bijou», etc. Pour mettre en valeur le produit décrit, les étudiants utilisent des entailles scripto-visuelles tels que les mots en majuscules, les mots en gras, les guillemets, les soulignements, l'écriture en couleur et en italique et les images du produit. L'objectif est d'attribuer à travers ces caractéristiques physiques «un statut» spécifique au produit décrit. La mise en valeur de ces éléments à la surface du texte améliore la lisibilité globale de la lettre et oriente le destinataire vers le but de la lettre.

Les mécanismes de cohésion nominale à propos de l'objet sont utilisés de manière satisfaisante dans notre corpus. En gros, la référence anaphorique est bien effectuée chez la presque totalité des apprenants même s'il y a des procédés répétitifs. En effet, des occurrences d'anaphores fidèles et des occurrences du pronom «il » ont été soulevées dans quatre lettres ; ceci crée une répétition qui peut 
apparaitre maladroite. Varier les reprises anaphoriques est important dans ce sens.

\subsubsection{Description de l'objet}

En ce qui concerne l'objet à promouvoir, les stratégies de valorisation par la mise en valeur des qualités techniques de l'objet et par l'emploi d'adjectifs positifs axiologiques sont généralement bien employées. Le problème se situe plutôt au niveau des arguments relatifs à l'objet qui devraient être mieux mis en ordre, mieux analysés pour être plus convaincants et persuasifs. En effet, l'absence de preuves concrètes (chiffrées ou autres) dans huit lettres rend la description des qualités techniques du produit générale et peu persuasive. Pourquoi le Sumsung SGH-E330 est-il léger ? Comment est sa forme ? Quel est son prix exact? Quelle est sa taille ? etc. En répondant à ces questions, le scripteur apportera des preuves qui donneront une force aux faits et rendront les arguments crédibles aux yeux des énonciataires. La preuve, comme dit Grize (1990: 44) est «le meilleur des arguments ». Elle donne à la lettre de vente sa force persuasive et assure le passage du CROIRE au FAIRE qui ne peut être garanti que si le client apprend quelque chose qu'il considère comme vrai et fiable.

La description subjective du produit est faite généralement par des adjectifs axiologiques et rarement par des adjectifs affectifs et des expressions de sentiment et d'émotion. L'usage des adjectifs axiologiques ne pose pas problème pour les étudiants. Tous ont utilisé des qualificatifs pour valoriser le produit. L'emploi des adjectifs affectifs, quant à lui, est remarquable dans trois productions. Il semble que les étudiants n'ont pas conscience du rôle de ce type d'adjectifs dans l'argumentation. Leur faible engagement affectif diminue la dimension émotionnelle de la lettre et porte atteinte à sa qualité argumentative. Pour persuader le client de l'intérêt du produit, l'énonciateur doit manifester ses réactions émotives et les faire partager et ressentir par le client. Son but est de produire un effet émotionnel sur le destinataire pour faire naître chez lui le désir d'achat. L'effet pathémique visé est marqué par la désignation lexicale 
de l'émotion qui renforce une adhésion et engage à l'action. La réussite de la stratégie argumentative dépend de l'adéquation émotionnelle entre l'énonciateur et l'énonciataire.

4.4. Quatrième phase : étude du dispositif d'énonciation

4.4.1. La prise en charge énonciative

Dans les productions des apprenants, la prise en charge des énoncés se fait en se présentant sous le nom de Maroc Telecom ou en utilisant le «nous» de l'entreprise. L'usage du «je», quant à lui, est exceptionnel. La prise en charge des énoncés par l'énonciateur est forte dans 27 lettres produites. La catégorisation du scripteur dans le statut social du responsable de la prospection et du Marketing ou sa présentation sous le nom de Maroc Telecom permet de resituer la communication écrite adressée dans un cadre professionnel conventionnel : il s'agit bien d'une communication écrite commerciale entre deux partenaires occupant des fonctions sociales précises et remplissant des fonctions bien déterminées.

\subsubsection{La référence au destinataire}

L'inscription de l'énonciataire dans la lettre se fait de différentes manières dans le corpus. Les pronoms d'adresse «vous» et les possessifs «vos/votre » sont utilisés par tous les étudiants avec des occurrences différentes. Les appellatifs (Monsieur, Cher Monsieur, Madame, Cher client, Chers clients, Mme, Mlle) sont employés par la totalité les étudiants pour désigner l'énonciataire. L'emploi des noms propres pour désigner le destinataire est, quant à lui, exceptionnel. Cette forme de désignation dénominative est présente dans trois lettres. Connaissant le destinataire, le scripteur s'adresse directement à lui par son nom. Ceci est un moyen efficace pour personnaliser sa lettre.

5. Conclusion et recommandations

Cette étude nous a permis d'identifier les principales difficultés des apprenants marocains en formation économique au supérieur dans un exercice particulier: l'argumentation dans une lettre commerciale de 
vente par correspondance. Certains problèmes relèvent de l'enseignement $\mathrm{du}$ français général (usage des connecteurs argumentatifs, enchaînement des arguments), tandis que d'autres touchent la formation en Communication de spécialité (implication du destinataire dans la lettre de vente, inscription de l'affectivité dans le discours commercial, appropriation de la structure compositionnelle de la lettre).

D'après nos constats, le degré d'investissement de nos étudiants dans l'écriture spécialisée ne se révèle pas important. En vue de développer leur compétence discursive en communication spécialisée en entreprise, il serait utile d'enrichir le contenu du programme de Communication en deuxième année par d'autres activités en français de spécialité: production d'articles économiques, de dissertations à partir de sujets économiques d'actualité, de textes publicitaires, de lettres commerciales à dominante argumentative à partir d'un spot publicitaire diffusé à la télévision ou d'une brochure qui décrit les qualités du produit, etc.

Pour développer la compétence argumentative des apprenants dans la formation donnée à l'École (cours de communication), il faudrait travailler davantage sur des extraits de magazines spécialisés, de journaux économiques et de revues de commerce. L'objectif est de faire acquérir des savoirs et savoir-faire en communication spécialisée qui pourraient être utilisés dans l'exercice de la profession.

Pour contribuer à résoudre les problèmes de langue rencontrés par les apprenants en formation économique, nous jugeons utile de faire travailler les étudiants sur l'analyse des textes en vue d'étudier leur structure et d'expliciter le fonctionnement des connecteurs, leur rôle au sein du discours et l'enchaînement des arguments qui déterminent la force argumentative globale de la lettre.

En outre, et en vue de développer la compétence linguistique et langagière des étudiants marocains arabisés, nous estimons essentiel de proposer aux nouveaux bacheliers admis à l'ENCG une formation de base en français en première année pour améliorer leur niveau en langue, corriger leurs lacunes et contribuer, par conséquent, à renforcer leur compétence textuelle en français de spécialité. 
Bibliographie

Adam J.-M. (1997) : L'Argumentation publicitaire. Rhétorique de l'éloge et de la persuasion. Paris : Nathan Université.

Adam J.-M. (1996): Les textes, types et prototypes: récit, description, argumentation, explication, dialogue. Paris : Nathan.

Adam J.-M. (1990): Eléments de Linguistique textuelle: théorie et pratique de l'analyse textuelle. Liège : Mardaga.

Amossy R. (2000) : L'argumentation dans le discours. Discours politique, Littérature d'idées, Fiction. Paris : Nathan.

Amossy R. (1999): Images de soi dans le discours. La construction de l'ethos. Genève : Delachaux et Niestlé.

Anscombre J.-C. (1995) : Théories des topoi. Paris : Éditions Kimé.

Anscombre J.-C. \& Ducrot O. (1983): L'argumentation dans la langue. Liège : Mardaga.

Bronckart J.-P. (1997): Activité langagière, texte et discours: Pour un interactionnisme socio discursif. Paris : Delachaux et Niestlé.

Bronckart J.-P. (1994): "Action, langage et discours. Les fondements d'une psychologie du langage », Bulletin suisse de linguistique appliquée, ${ }^{\circ} 59$, pp.746.

Charolles M. (1995) : «Cohésion, cohérence et pertinence du discours », Travaux de Linguistique, $\mathrm{n}^{\circ} 29$, pp.125-151.

Ducrot O. (1980) : Les échelles argumentatives. Paris : Éditions de Minuit.

Everaert-Desmedt N. (1984): La communication publicitaire. Étude sociopragmatique. Louvrain-La-Neuve : Cabay.

Grassi M.-C. (1998) : Lire l'épistolaire. Paris : Dunod.

Holtzer G. (2004) : «Savoirs et compétences en français-écrit d'élèves guinéens : les enquêtes CAMPUS », Le français en Afrique, ${ }^{\circ}{ }^{\circ}$ 19, pp. 37-73.

Maingueneau D. (1996) : Les termes clés de l'analyse du discours. Paris : Seuil.

Kerbrat-Orecchioni C. (1986): L'énonciation et la subjectivité dans le langage. Paris : Armand-Colin.

Maingueneau D. (1998) : Analyser les textes de communication. Paris : Dunod.

Perelman C. \& Olbrechts-Tyteca (1970), 1ère éd. (1958) : Traité de l'Argumentation. La nouvelle Rhétorique. Bruxelles : Éditions de l'Université de Bruxelles.

Peytard J. (1982) : Littérature et classe de langue. Paris : Hatier-Crédif. 